\title{
Snhaltäverzeidhis.
}

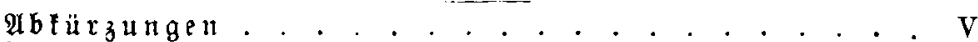

Tert ber (3) efebesoorforiften über bie Semährleiftung megen Mängel ber ๔ad)e (\$\$ 459-492 $\mathfrak{B}(3) \mathfrak{B}$.) unb bie Berorbnung über \$auptmängel voun 27. März 1899

\section{I. $\mathfrak{A} \mathfrak{b}\{\mathfrak{d} \mathfrak{u} i t \mathfrak{t}$.}

\section{Dic allgemeiuen Medtsooridrijten.}

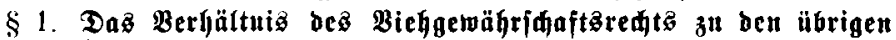
Boridrifteit

I. Die Borfariften beș $\mathfrak{B}$

II. Das Sanbelsgejebbud) . . . . . . . . . . 12

III. Marttoronungen uno ธandelzgebräude . . . . . 13

IV. Srtliche Statutentortiiton . . . . . . . . . 15

§ 2. Die Infeđ1tutg wegen $\mathfrak{Y r r t u m}$. . . . . . . . . . . . 16

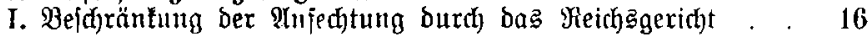

II. Begriff ber verfehratwelentlichen (Eigenichajten . . . . . 16

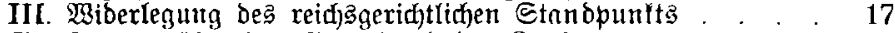

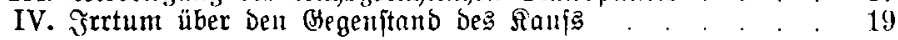

\$3. Die Injedtung wegen arglifitger $\mathfrak{x a ̈ l f d u n g ~ . ~ . ~ . ~ . ~ . ~ . ~ . ~ . ~} 20$

I. Irgfiftige Täuf́hıng . . . . . . . . . . . 20

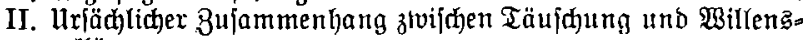
ertlärung

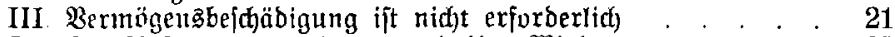

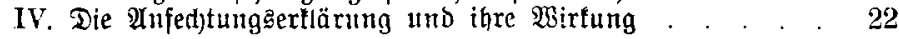

§ 4. Argliftiges $\mathfrak{B e r i d w e i g e u ~ . ~ . ~ . ~ . ~ . ~ . ~ . ~ . ~ . ~ . ~ . ~ . ~ . ~ . ~ . ~} 22$

I. Unterbrüđung eirca Mangels . . . . . . . . . . . . 22

II. Blopes Berfatweigen bei Sffenbarungapilidyt : . . . . 23

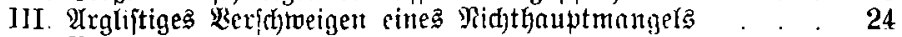

IV. Beweislajt . . . . . . . . . . . 26

§. Berifulton bei (Fingehung beş Bertrag ż . . . . . . . . . 26

\$ 6. Beridulban bei Erfiillung bes Bertrags . . . . . . . . . . 29

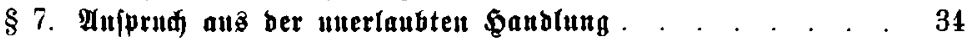

\$ 8. Roulurrenz ber mefreren $\mathfrak{A n}$ fprüde . . . . . . . . . . . . 36

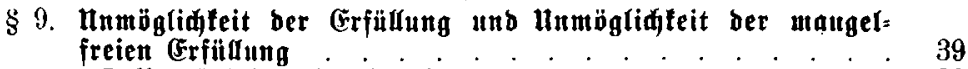

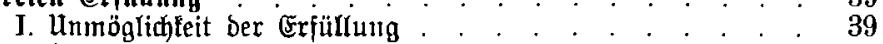

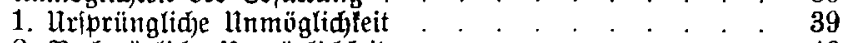

2. Nađ̆trägliçe Unmögliđłłeit . . . . . . . . . . . . . . 40

II. Sunlitative unmöglidfeit. . . . . . . . . . . . . . . . $\quad .40$

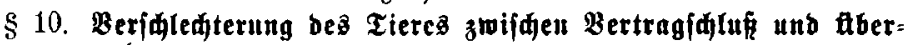
gabe 


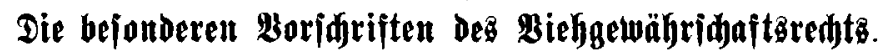

§ 11. Die privilegierten Tiergattutgen . . . . . . . . . . . . . 47

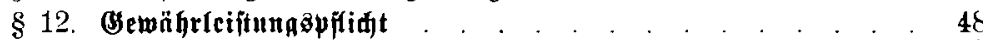

I. Semäl)rlestung . . . . . . . 48

II. J)ä̧gebender Beitpunft für bie Be[d]afjenheit ber Stauffache 50

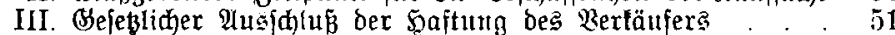

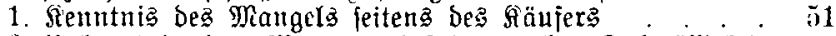

2. Hnfenntuis bes MRangels infolge groker Fahrlälligteit bes $\Re$ äufers

3. Borbehaltlole giuname

4. Lerfäumuntg ber Mängełanzeige $\quad 57$

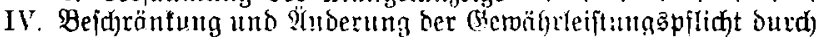
Bereinbarung

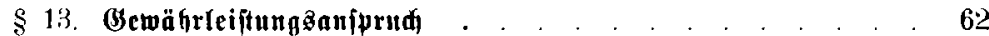

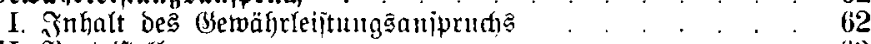

II. \$̧arteifteIlung . . . . . . . 63

1. Mftiolegitimation . . . . . . . . . . . 63

2. \$affiulegitimation .56

\$ 14. Gauptmängel . . . . . . . . . . . . . . . . . . . . 67

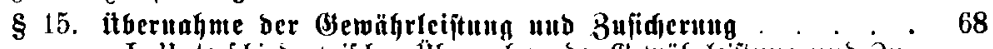

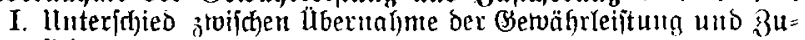
fidjerung . . . . . . . . . 68

II. Erforberniffe der 3ujicherning einer Figestichait . . . 71

1. Bertragsెmäß̈gleit ber ßu[idjerung . . . . . . . . 71

2. Eigenihaftent . . . . . . . . . 77

III. Einzelfälle von Eigenịhaften $. . \quad . \quad . \quad . \quad . \quad . \quad . \quad .82$

IV. Unteridiebliche Regelung, je nachdein eine (Setwähririft ver= einbart ift ober nidht . . . . . . . . 83

§ 16. Itbergang ber (5efahr . . . . . . . . . . . . . . . 83

I. Bebeutung bes Mejahrübergangs . . . . . . . . . . . . $\quad .83$

II. Beitpuntt deß̉ Gejahrübergangş.

1. Ulbergabe . . . . . . . . . . . . . . . . . 84

2. Serfendung . . . . . . . . . . . . . . . . 85

3. Rnualmeverzug

4. Bevingter fauf . . . . . . . . . . . . . 87

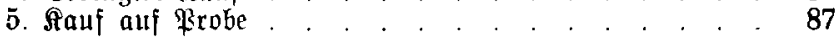

\$17. Bewährfilit . . . . . . . . . . . . . 88

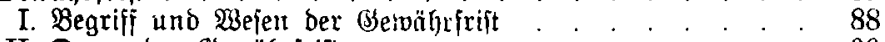

II. Dauer ber B́wewährfrift

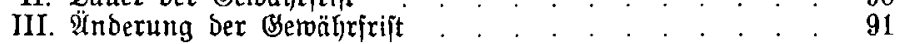

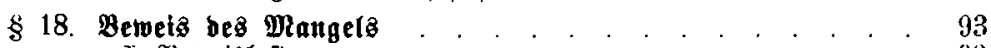

I. Belveišlaft . . . . . . . . . . . . . . 93

II. Betweißfilfrung

1. Went eine Bewährfrift anzurbenden ift . . . . . . . 94

a) bei ફ̧auptmängeln

b) bet anderen Mängeln

2. Wenn teine Betwährfrift anzuvenden ift . $\quad . \quad . \quad . \quad .98$ 


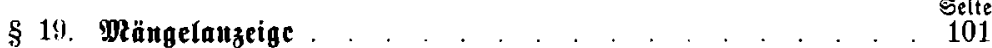

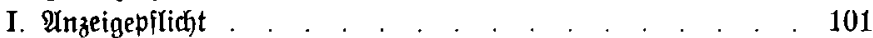

1. Bezeidnung des Martgels . . . . . . . . . 101

2. थ̂Azeige deš fiäluerz . . . . . . . . . . . . 103

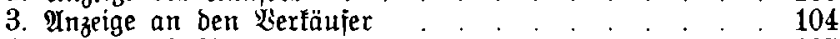

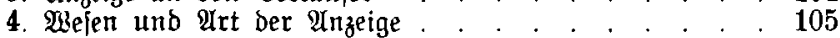

5. Die ber Unzzeige gleidytehenden Rechtshandungen . 106

6. 2̂njeigefrift 108

II. Folgen bex Beriäumung ber Arnzetge . . . . . . . . . . 109

III. $\mathfrak{B}$ iberruf ber Mängelanzeige

IV. Reine Unzeigepflicht bei argliftigem Kerfuneigen bes $\mathfrak{B e r}=$ fäufers . . . . . . . . . . . . . . . . . . 110

V. Statutenfortifion

\$ 20, Wandrung . . . . . . . . . . . . . . . 111

I. Wejen ber Wandlung . . . . . . . . . . . . . . 111

II. Borauşęunget ber Wandlung . . . . . . 114

III. Zuläffigteit ber $\mathfrak{B a n}$ ólung bei untergang, Serichlechterung, Ehlahtung und Beräukerung bes Tieres

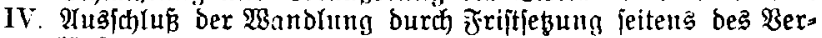
łäufers

V. Bollziehung ber Wautolung . . . 119

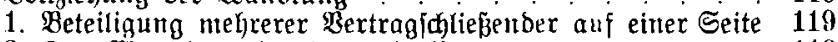

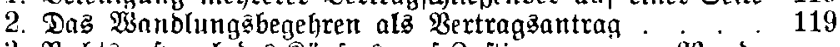

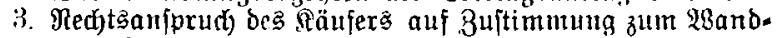
lungshegehren

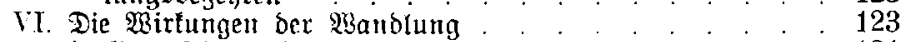

1. Gegenjeitige Rüđgerährpflicht . . . . . . . . . 124

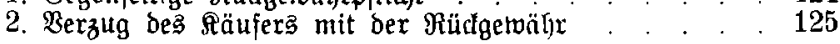

§ 21. Wanblung bei Mangelfaftigfeit nur einer vou ntehrerelt

Aauffaden . . . . . . . . . . . . . . 125

I. Brunbiäbritiłe Befdräntung ber Wlandlung auf die mangelhafte Sache. Entjpredtende Serablebung des GSejamtpreifes 125

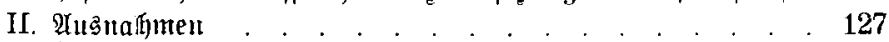

1. Saupt= uno Rebeniache

2. Wir tfanaftliche Intrembarteit . . . . . . . . . 127

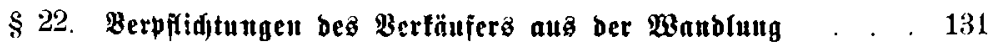

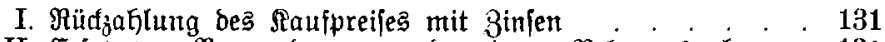

II. Erjaß von Berwendungen und anderen Rebenaugaben 131

1. Berwendungen . . . . . 131

a) Rojten Der Fütterung und Bflege . . . 131

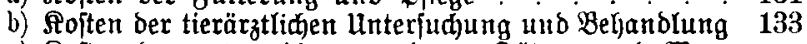

c) Poften ber notwenbig getworberten Tötung und $\mathfrak{B e g}^{2}$ ichaffung . . . . 134

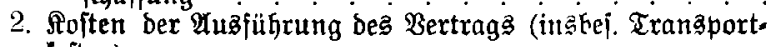
tofteil)

3. Foften eines Borprozefies . . . . . . . . . . 135

4. Bertragafoften

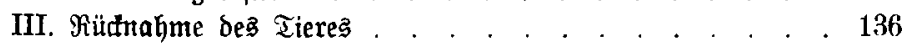


IV. Totten eines Bemeisficherungaverfokrenz

136

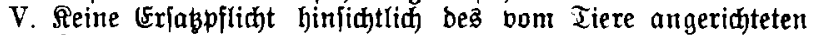
Sqabenż . . . . . . . . . . . . . . . 136

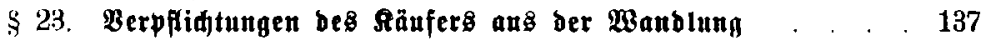

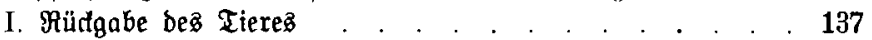

1. Rüđgabe in natura . . . . . . . . . . . . . 137

2. Intergang ober Berjhlehterung bes Tieres $: 137$

a) vor Bollziehung ber Mantlung . . . . . . . . 137

(c) infolge 8 ufalla (2̂nfprud) auf Bंerficherungajumme?) 137

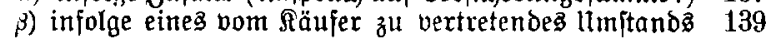

b) ntad) $\mathfrak{B o l l}_{\text {z }}$ ieljung ber Wandlung . . . . . . . 143

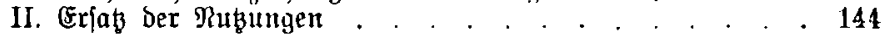

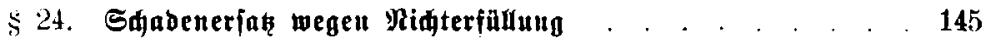

I. Die \$̧aftunģ̊gründe . . . . . . . . . . . . . . . 145

1. Bertragßmähige 8ufiderung . . . . . . . . 146

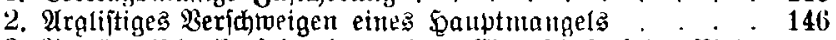

3. Betritgerijhe Boripiegelung einer (Eigenfdoft beim Bieh= fauf nicht aubreidhend . . . . . . . . . . . 146

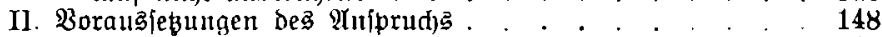

1. Borhandenfein des Mangels zur Zeit Des stauts . . 148

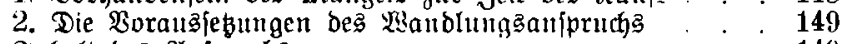

III. Эnhalt bes थnipruchs . . . . . . . . . 149

1. Ter 2upprud bejteht nidgt u e be u ber พBanolung 149

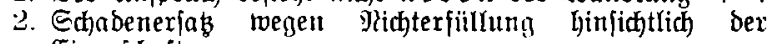
(Eigenid)aft . . . . . . . . . . 150

a) Umfang bes @djabenz . . . . . . . . . . . 150

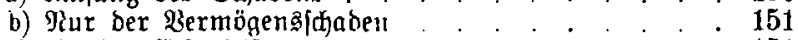

c) Prt ber Erjałleiftung . . . . . . . . . . . . . 151

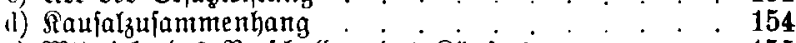

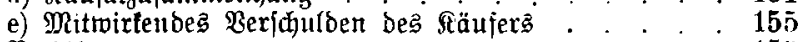

3. \$erjährung. . . . . . . . . . . . . . 156

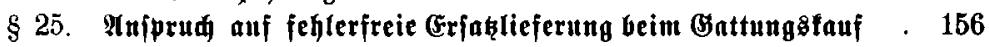

I. Spezies = und Battungałchulb . . . . . . . . . . 156

II. Die allpemeinen ßemährleî́tungäaniprübe . . . . . 156

III. Der befondere 2tnfprud auf Erjableiftung.$: 157$

IV. Dả Erforberniz Der mittleren $\mathfrak{A}$ rt und Büte beim

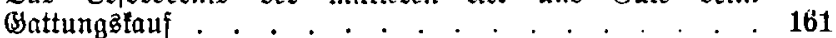

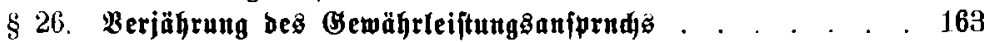

I. BetoüGrleifturtgåanprüde . . . . . . . . . . . . 163

1. Unipruch auf æanblung . . . . . . . . . . . 163

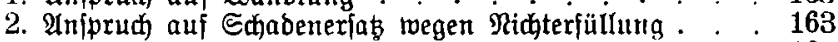

3. 2 miprud auf mangelfreie Erjab̧lieferung. . . . . . 164

II. Ũnbere Aniprïche . . . . . . . . . . . . . 164

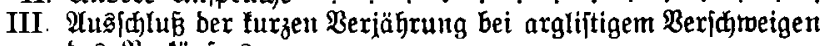
bes Bertäufer̉ . . . . . . . . . . 165

IV. Dauer Der Serjährungaffrift

1. Beginn

a) nă bem Geles . 166

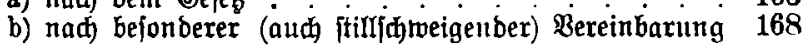


2. Ende.

3. Semmung und Unterbred)ung . . . . . . . . . 169

V. Wirfungen ber Berjährung . . . . . . . . . . . 172

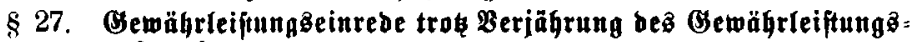
ariprudez

I. Boraubiegungen , . . . . . . . . . . . . . . 173

II. Einreberedht . . . . . . . . . . . . . . . . 175

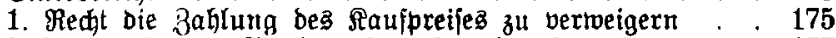

2. Recht mit bem Shabener\{abanfprud aufzured)nent : 177

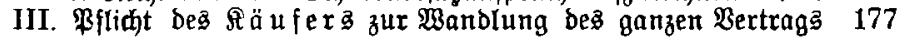

$\$ 28$. Berträge, bie auf Beränkerung ober Belaîtung einer Sađ̆e gegen Gutgelt geridet find . . . . . . . . . . . . 178

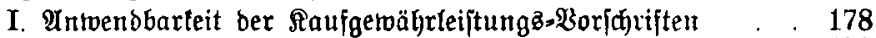

1. Taufd . . . . . . . . . . . . . . . . 178

2. 5ingabe an 3 ahlunga statt . . . . . . . . . 183

3. Rerjteigerung . . . . . . . . . . . . . . . 183

4. Bergleid

5. Teilung einer B̧emeinidajt . . . . . . . 184

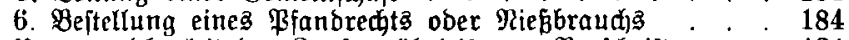

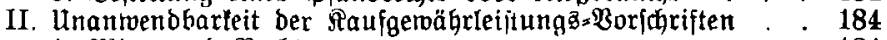

1. Miete und Pacht . . . . . . . . . . . . . . 184

2. Eeifuertrag . . . . . . . . . . . . . . . . 184

3. Sdgentung . . . . .

4. $\mathfrak{A}$ sftattung . . . . . . . . . . . . . 185

5. Bermtädtutį . . . . . . . . . . . . . . . . 185

6. Brangsenteignung . . . . . . . . . . . . . 185

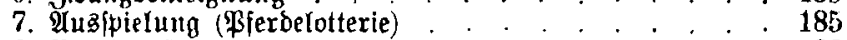

8. Pfanbveriteigerung . . . . . . . . . . 186

9. Anoronung Der Serfteigerung burdi ben Ridter . . . 187

\$ 29. Wrozekéredtlide $\mathfrak{F r a g e n ~ . ~ . ~ . ~ . ~ . ~ . ~ . ~ . ~ . ~ . ~ . ~ . ~ . ~ . ~} 188$

I. 8 ự änbigfeit

1. Sachlide ßuftändigleit . . . . . . . . . . . . . . 188

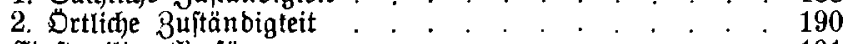

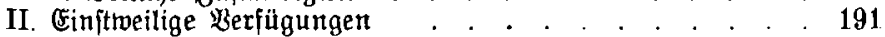

1. Serfteigerung bez Tiere? . . . . . . . . 191

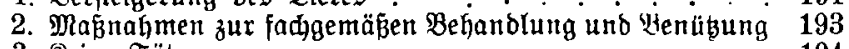

3. Reine Tötung. . . . . . . . . . . . 194

III. Frozeß̉toften . . . . . . . . . . . . . . . 194

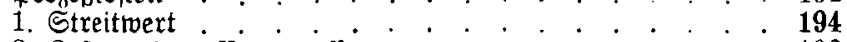

2. Roîten eines Borprozeffes . . . . . . . . . . . . . . 196

3. Sloften eines Beweişicherungoberfahrens. . . . . . . 196

4. Roften einer einjtweiligen Berfügung . . . . . . . 196

III. $\mathscr{U} b\lceil\boldsymbol{q}$ it $t$.

\section{Der Gauptmängel=Satalog Der Raiferliden Berorouung.}

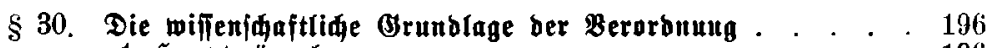

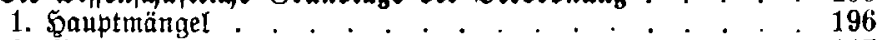

2. Setwährfriften . . . . . . . . . . . . . 197 


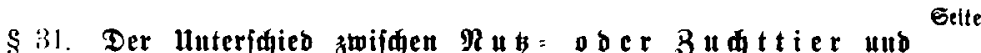

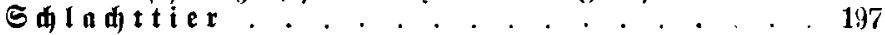

8 32. Şauptmängel ber Pferbe, Gjel, Dlauleiel und Miaulticre. . 202

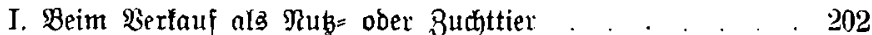

1. Roł (खurm) . . . . . . . . . . . . . . 202

2. Dummfoller (Foller, Tummfeir) . . 203

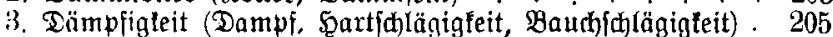

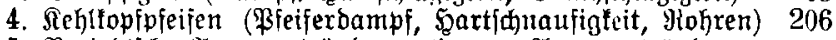

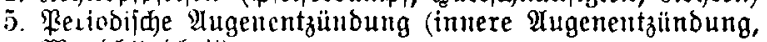
Monbblindheit) 208

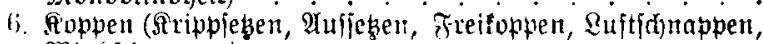
Wintidfuppen

II. Beim Sertauf ale હdlađtitier . . . . 210

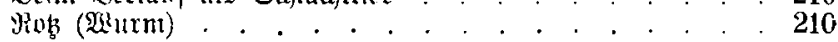

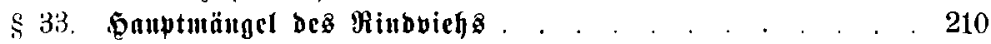

I. Beim Berfauf als $\mathfrak{R u b}_{0}$ oder Guthttier . . . . . . . 210

1. Tuberfubje Extraufung . . . . . . . 210

2 Sungenfeudje . . . . . . . . . . 212

II. Beim Serfauf als sdl(ad)twieh) . . . . . . 213

Tubertulöie Erfrantung . . . . . . . . . . . . . . . . 213

\$ 34. bauptmängel ber Saafe . . . . . . . . . . . . . . . 214

I. Beim Bertauf ala Pung= ober Burbttier . . . . . . . 214 Räude . . . . . . . . . . . . 214

11. Beim Ber"auf als Shladttier . . . . . . . . . . 215

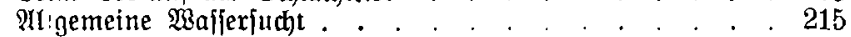

\$ 35. Sauptmäugel ber @dweiue . . . . . . . . . . . . 215

I. Beim Berfauf als Rubo ober Zudttier . . . . . . . 215

1 Rotlauf . . . . . . . 215

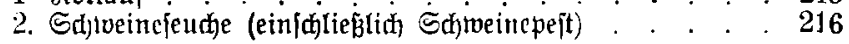

II. Beim Bertauf alz Shladttier . . . . . . . . . . . 216

1. tuberfulöfe Erfrantung. . . . . . . . . . . . . . 216

2. Tridjinen . . . . . . . . . . . . . . . . 216

3. Finnen

IV. $\mathfrak{A} \mathfrak{b} \mathfrak{d} \mathfrak{d}) \mathfrak{i t}$ t.

Mligtbauptmängel แnd Mtangel zugefidjerter Bigenjaaft.

§ 36. feglerfreifheit . . . . . . . . . . . . . . . . . . . 217

I. Senftung im allgemeinen . . . . . . . . . . . . . . . . . 217

1. Relatiber $\mathfrak{B}$ egriff . . . . . . . . . . . . . . . . . 217

2. Borübergehende $\mathfrak{M}$ ängel uno sirantheiten . . . . 218

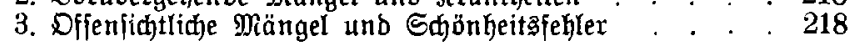

II. Gaftung im bejonberen . . . . . . . . . . . . . . . . . 219

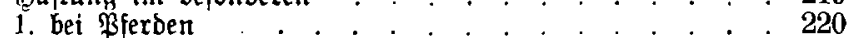

a) dhronifhes Srummgehen : . . . . . . . . . . 220

b) Mangel Der Frömmigleit . $\quad . \quad . \quad . \quad . \quad . \quad . \quad . \quad . \quad . \quad .220$

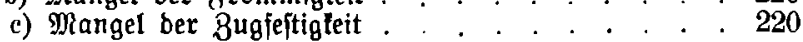


d) habituelles ভtolpern . . . . . . . . . . . 220

e) Badtltrab . . . . . . . . . . . . . . . 220

f) Meben (Srippengeigen) . . . . . . . . . . . . 220

g) Räube . . . . . . . . . . . . . . . 220

h) Maufe . . . . . . . . . . . . . . . . . 220

i) פibaublalag

k) Segen und arlffteha . . . . . . . . . . . 221

1) fibledtex Freifer (Sdjieferzaln, Scherengebißj) . . . 221

in) IIter . . . . . . . . . . . . 229

n) Breifen (Einfauen ber tointereifest in Die Borbereifen) 222

o) Streifen . . . . . . . . . . . 222

1) 3itgellahmbcit; furzer 官ritf; sabnentritt . . . 222

q) Wehler an Den Tugen . . . . . . . . . . . . 222

r) (Sanajderztwang . . . . . . . . . . . . 222

s) Inermüuld)te Träd)tigle it . . . . . . . . . . . . . . 222

t) Inftedenbe Frankheiten , . . . . . . . 223

a) Ehtoindel und Epilepfie . . . . . . . . . . 223

v) Startframpf (Tetanus-Bazillus) . . . . . . . . 223

2. Bei Minboieh . . . . . . . . . . . . . . . 223

a) Edjeibeborfall . . . . . . . . . . . . 223

b) Edbeidenfotarrh . . . . . . . . . . . . . 223

c) Etrahlpil $z=$ Erfrantung $\quad . \quad 224$

d) 3urüđbleiben ber Rad)geburt (Meinfein) . . . . 224

e) Hitillerfrautheit (Stierfud)t: . . . . . . . . . 224

f) $\mathfrak{M}$ ifdutuh

g) Wiberfeblic)leit . . . . . . . . . . . . . 224

h) Mangel ber 3ugfeftigfeit : . . . . : 224

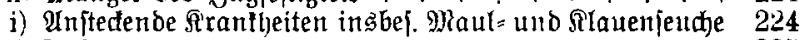

k) Tuberiulofe . . . . . . . . . . . . . . 220

l) Entzündung Durh Frembförper . . . . . . . . . 225

3. Bei Schafen . . . . . . . . . . . . . . . 226

t. Bei Srbweinen . . . . . . . . . . . . . . 226

§ 37. Befunbqeit . . . . . . . . . . . . . . . . . . 228

\$38. Seudenfreiheit . . . . . . . . . . . . . . . . . . . 229

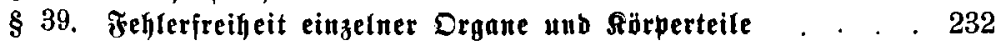

I. Bute Beine . . . . . . . . . . . . . . . . 232

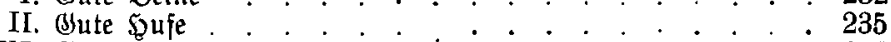

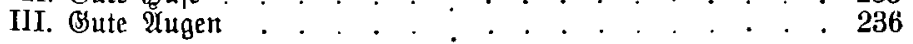

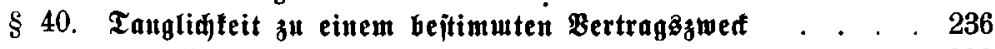

I. „Butes Reitpferd"; ,gute Sdhubfuh". . . . . . . . . 236

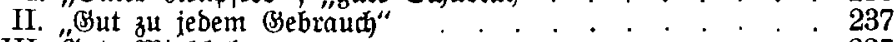

III. "ß゙ute Mild)

a) IIIgemeine

b) ₹rrifđmilçen (

c) Betrügerijhes Euterjpannen : . . . . . . . . . . . . 239

IV. 3 udttauglidfeit . . . . . . . . . . . . . . . 240

\$ 41. Bugfeffigfteit . . . . . . . . . . . . . . . . . 241

§ 42. Frömmigfcit . . . . . . . . . . . . . . . . . 244 
\& 43. Trähtigfeit ......... T49

I. Begriff Der Träd)tigteit . . . . . . . . 249

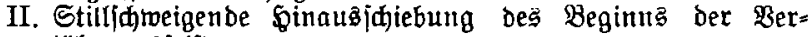
jährunģ̧⿻三丨ift . . . . . . . . . . . . . . . 249

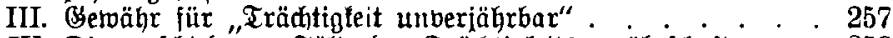

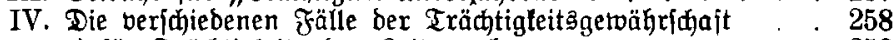

a) für Trüd)tigfeit ofne Zeitangabe . . . . . 258

b) für Trädttigteit von beftimmter Dauer . . . . . . 259

c) für $\mathfrak{W e r f e n ~ z l l ~ e i n e m ~ b e f t i m m t e n ~ B e i t p u n f t ~ : ~ . ~ . ~} 260$

d) für "ein Ralb" („ein Fohlen") ohnte Beitangabe : . 263

e) für "eint $\mathfrak{S a l b}^{\prime \prime}$ (",ein Froflen" bis zul einem beftimmten 3eitpuntt)

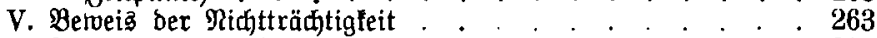

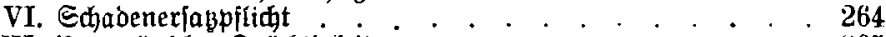

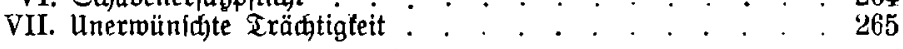

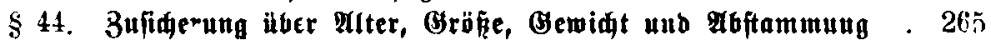

